\title{
Why Hurricane Categories Should Be Based on Surface Pressure
}

Adapted from "Surface Pressure

A More Skillful Predictor of Normalized Hurricane Damage Than Maximum Sustained Wind," by Philip J. Klotzbach (Colorado State University), Michael M. Bell, Steven G. Bowen, Ethan J. Gibney, Kenneth R. Knapp, and Carl J. Schreck III. Published online in BAMS, June 2020. For the full, citable article, see DOI:10.1175 /BAMS-D-19-0062.1.

A tlantic hurricanes in 2017 and 2018 resulted in more than $\$ 345$ billion in damage for the United States and Caribbean. The primary drivers of the damage varied significantly for each individual stormfrom freshwater-driven impacts for Harvey and Florence to surge- and wind-driven effects for Irma and Michael. While no singular geophysical quantity can perfectly explain the damage a storm will cause, here we compare how maximum sustained wind speed $\left(V_{\max }\right)$ and minimum sea level pressure (MSLP) perform at predicting hurricane damage in the continental United States (CONUS).

The Saffir-Simpson hurricane scale, originally designed by Herb Saffir to reflect wind damage, was first presented in 1969. It ranked hurricane wind damage on a 1-to- 5 scale, with 5 indicating the strongest winds and the greatest damage. Robert Simpson added MSLP and typical storm surge values associated with each hurricane category. The Saffir-Simpson hurricane scale was used by the National Hurricane Center (NHC) to convey the multiple threats from hurricanes for more than 30 years. However, costly and large hurricanes like Katrina (2005) and Ike (2008) brought more storm surge than would have been expected given their Saffir-Simpson categories at the time of landfall. In response, NHC removed storm surge and MSLP from the scale and now uses the Saffir-Simpson hurricane wind scale (SSHWS) based on $V_{\max }$ alone in public warnings. 
MSLP is recorded consistently in the definitive Atlantic hurricane database, HURDAT2, for all named storms since 1979 and CONUS landfalling hurricanes since 1851. Given the strong relationship between MSLP and $V_{\max }$, use of MSLP as the primary intensity metric would reduce some of the challenges of analyzing global or regional TC intensity trends.

MSLP is much more easily identified than $V_{\text {max }}$, which, according to the NHC, is nearly impossible to measure directly. The reference winds are at $10 \mathrm{~m}$ with an open exposure, which happens at sea where there are fewer observations. Likewise, the maximum winds in a hurricane are extremely difficult to measure at any particular time. Conversely, the definition of MSLP is clear: it is at the center of the storm and is part of the large-scale vortex structure. MSLP is therefore relatively easily located and is already very accurately measured with aircraft reconnaissance, especially for strong hurricanes.

MSLP is much easier to measure at landfall than $V_{\max }$. Anemometers are prone to mechanical failure at wind speeds above $50 \mathrm{kt}$, precisely when they matter most. One recent simulation study showed that even a perfectly positioned, operating anemometer in the right-front quadrant of a hurricane would underestimate the actual storm's intensity by $10 \%-20 \%$, on average. This study also noted that a barometer in the eye of a hurricane is likely to overestimate MSLP by only $1-3 \mathrm{hPa}$.

The central pressure deficit (the difference between the pressure of the surrounding largescale environment and the MSLP) increases not only with $V_{\max }$ but also with storm size and background rotation rate. While acknowledging that changes in both the environmental pressure and the Coriolis parameter are not negligible, here we focus on MSLP as our primary intensity metric to compare with $V_{\max }$.

MSLP is effectively an integrated metric of the entire wind field, which affects storm surge. Increased storm size, especially for major hurricanes over mildly sloping coastal shelves-such as along the Gulf of Mexicoalso increases wind and rainfall footprints. Consequently, as a single metric, MSLP provides more information about potential hurricane damageability than $V_{\max }$.

Saffir-Simpson hurricane scale with current $V_{\max }$ criteria, proposed MSLP criteria, and original MSLP criteria from 1974. Also provided in parentheses are the percentage of Atlantic storms from 1979 to 2018 whose lifetime maximum intensity exceeded the weakest intensity criteria for each category threshold.

\section{Data and methodology}

We examined a long historical record (19002018) of normalized CONUS hurricane damage to evaluate if $V_{\max }$ or MSLP has historically provided a better estimate of hurricane damage. The normalization translates historical hurricane damage to the hypothetical damage these storms would cause now given today's population, inflation, and per capita wealth.

We employ data from 165 CONUS hurricane landfalls from 156 hurricanes from 1900 to 2018. Though Hurricane Sandy officially became post-tropical several hours before landfall, we include it given the tremendous physical and humanitarian impacts that the storm caused. While the estimated maximum winds after landfall were $65 \mathrm{kt}$ (e.g., category 1), Sandy's landfall MSLP of $942 \mathrm{hPa}$ would equate to a category 4 hurricane on the original Saffir-Simpson scale.

Both $V_{\max }$ and MSLP for hurricanes at landfall in the CONUS are based on HURDAT2, which has all U.S. landfalls identified from 1851-1960 and from 1983-2018. For 1961-1982, we used the CONUS hurricane impacts database from the Atlantic Oceanographic and Meteorological Laboratory, since data during this period in HURDAT2 do not directly identify landfalls. This impacts database only provides the $V_{\max }$ and MSLP intensity at the time of a hurricane's strongest landfall, as opposed to HURDAT2, which provides intensity information for all hurricane landfalls. Nine hurricanes in our landfalling hurricane dataset had MSLP calculated from a $V_{\max } /$ MSLP relationship as opposed to a direct measurement. Removing these nine hurricanes from our analysis does not significantly change our results.

\section{Results}

We examined the relationship between normalized hurricane damage and $V_{\max }$ and MSLP for 1900-2018. The rank correlation between MSLP and normalized damage $\left(r_{\text {rank }}=0.77\right)$ 
(a)

Continental US Normalized Hurricane Damage Rank vs. Landfall $V_{\max }$ Rank (1900-2018)

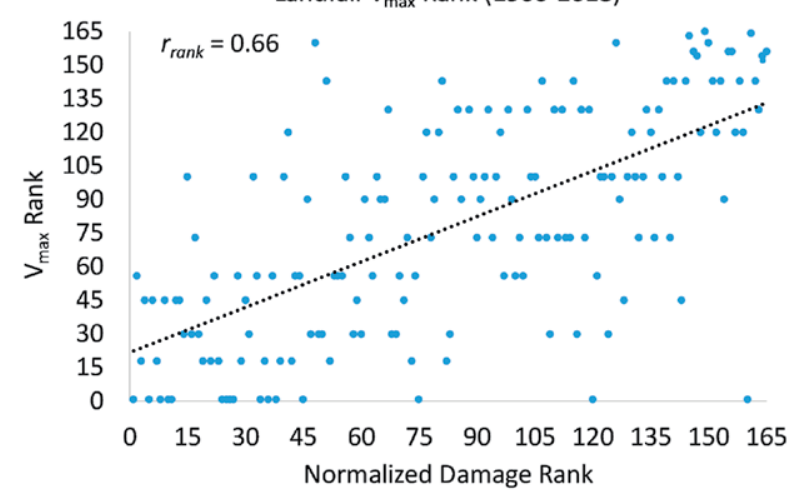

(c)

Continental US Normalized Hurricane Damage Rank vs. Landfall V $V_{\max }$ Rank (2007-2018)

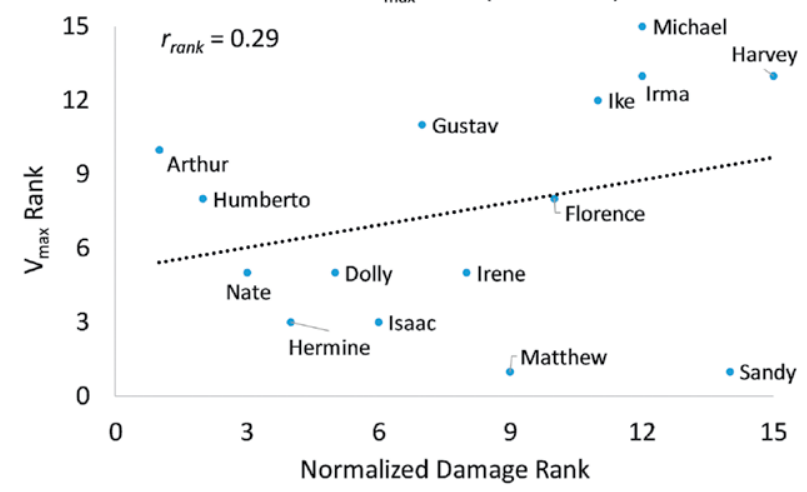

(e)

Georgia to Maine Normalized Hurricane Damage Rank vs. Landfall $V_{\max }$ Rank (1900-2018)

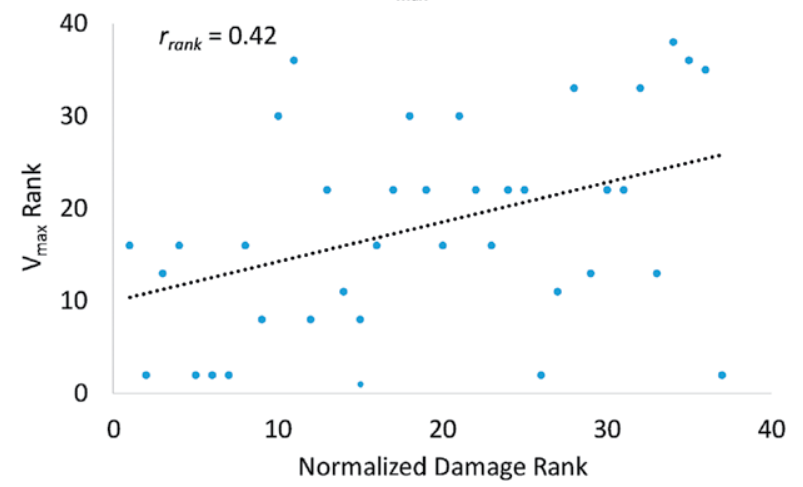

(b)

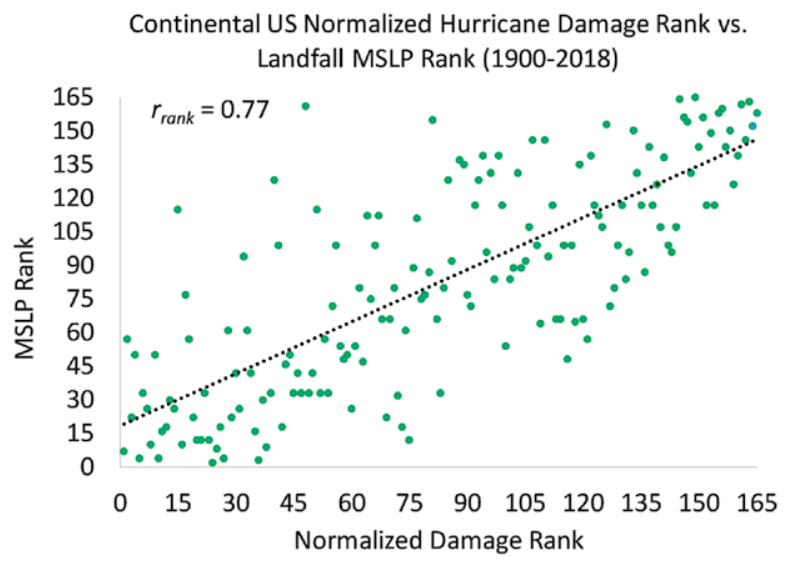

(d)

Continental US Normalized Hurricane Damage Rank vs. Landfall MSLP Rank (2007-2018)

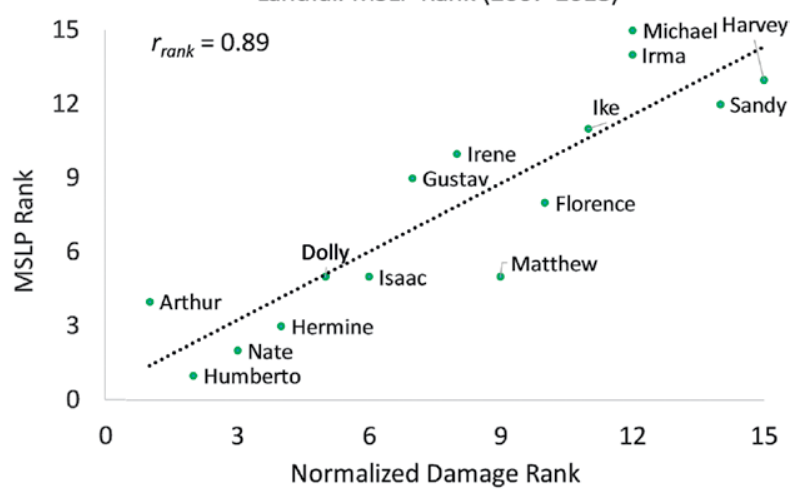

(f)

Georgia to Maine Normalized Hurricane Damage Rank vs. Landfall MSLP Rank (1900-2018)

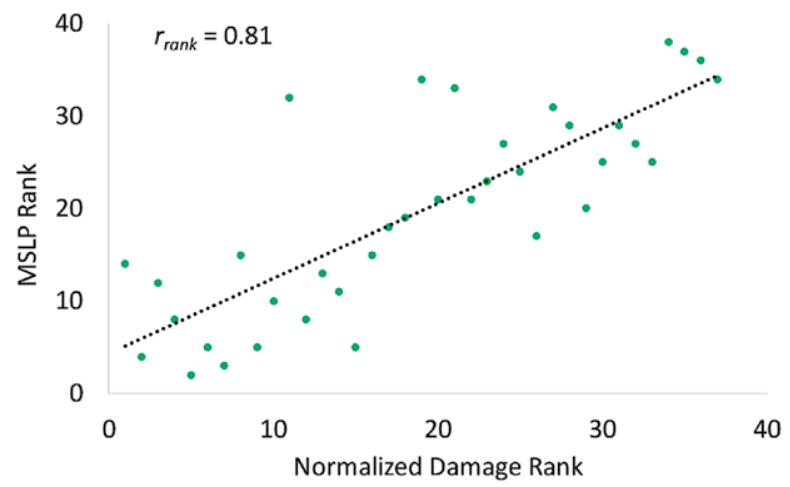

is significantly greater than that for $V_{\max }$ $\left(r_{\text {rank }}=0.66\right)$.

While both quantities correlate significantly with normalized damage, damage is more than a function of the intensity of the TC. Damage is also driven by the size of the exposed population. In addition, slow-moving TCs like Hurricane Harvey (2017) and Hurricane Florence
$\Lambda$ * Normalized hurricane damage relationship with $\boldsymbol{V}_{\max }$ and MSLP. (a) For CONUS normalized damage vs $V_{\max }(1900-2018)$, (b) for CONUS normalized damage vs MSLP (1900-2018), (c) for CONUS normalized damage vs $V_{\text {max }}$ (2007-18), (d) for CONUS normalized damage vs MSLP (2007-18), (e) for Georgia to Maine normalized damage vs $V_{\max }(1900-2018)$, and (f) for Georgia to Maine normalized damage vs MSLP (1900-2018). Individual CONUS hurricane landfalls are labeled on (c) and (d). For these plots, higher ranks indicate greater $\boldsymbol{V}_{\max }$ (or lower MSLP) and increased levels of normalized damage. 
(2018) illustrate the strong relationship between freshwater flooding and damage. Consequently, we cannot expect one TC intensity metric to correlate perfectly with damage.

In recent years, MSLP has shown much improved skill at predicting normalized damage when compared with $V_{\max }$ for landfalling CONUS hurricanes. From 2007 to 2018, the rank correlation between $V_{\max }$ and normalized damage decreased to 0.29 , while the rank correlation between MSLP and normalized damage increased to 0.89 . Both Hurricane Ike (2008) and Hurricane Sandy (2012) had much lower MSLP (Sandy: $942 \mathrm{hPa}$; Ike: $950 \mathrm{hPa}$ ) than would be expected for their SSHWS category during landfall. Given the extremely large size and associated energy these hurricanes had, both generated large and impactful storm surges.

We hypothesize that the tendency of hurricanes to grow as they move poleward may weaken the relationship between $V_{\max }$ and normalized damage for storms making landfall from Georgia to Maine. The 1900-2018 correlation between MSLP and normalized damage is significant and strong for Georgia to Maine landfalls $\left(r_{\text {rank }}=0.81\right)$, while the correlation for $V_{\text {max }}$ is much weaker $\left(r_{\text {rank }}=0.42\right)$. Storm surge is related to hurricane size, which may help explain why MSLP is a better overall predictor of damage. Correlations for both $V_{\max }$ and MSLP with normalized damage are strong for Gulf Coast landfalls.

There would also generally be more confidence in identifying the landfalling SaffirSimpson category using MSLP than in using $V_{\max }$. The SSHWS categories based on $V_{\max }$ range from 13 to $24 \mathrm{kt}$, and the average $V_{\max }$ uncertainty is $9.8 \mathrm{kt}$ for CONUS landfalling major hurricanes. The revised MSLP ranges underlying those categories are $15-20 \mathrm{hPa}$, while the average MSLP landfalling major hurricane uncertainty is an estimated $3.6 \mathrm{hPa}$. With uncertainties so much narrower than the category ranges, we can therefore say with much greater certainty the exact category of a system when using MSLP. NHC adds uncertainty by rounding $V_{\max }$ values to the nearest $5 \mathrm{kt}$, while MSLP is rounded to the nearest $1 \mathrm{hPa}$.

In the normalized hurricane dataset since 1900, there are $67 V_{\max }$-defined CONUS major hurricane (category 3 or higher) landfalls, which generated $81 \%$ of the $1900-2018$ normalized hurricane damage. Using the MSLP-
Percentage of all Normalized Hurricane Damage Events Classified as Major Hurricanes using Vmax and MSLP Threshold

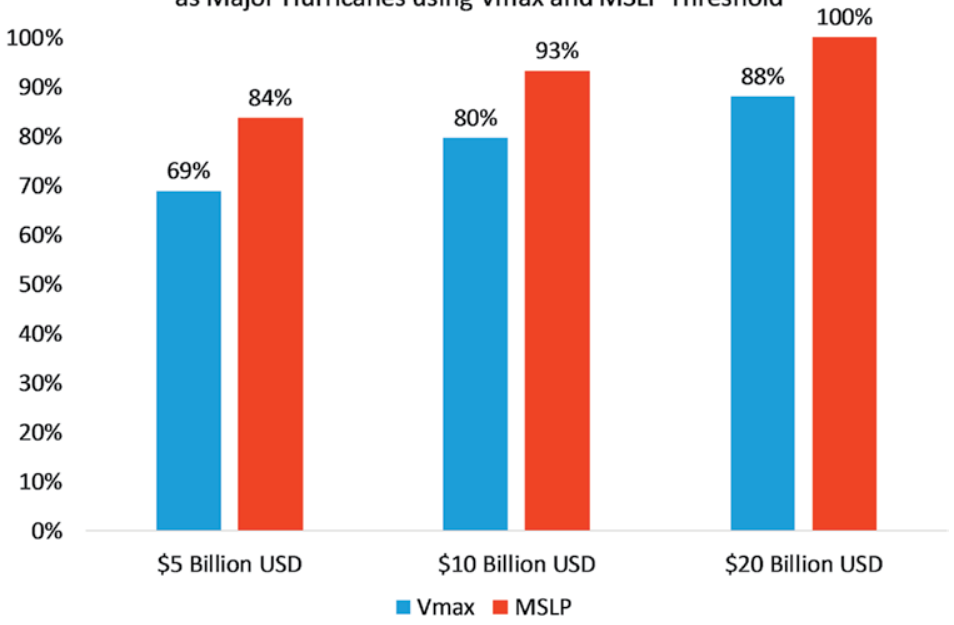

based definitions of major hurricane landfalls ( $\leq 960 \mathrm{hPa})$, there would have been 80 CONUS major hurricanes, yielding $92 \%$ of the normalized hurricane damage. Only two hurricanes would be downgraded using the MSLPbased definition: Storm 5 (1945) and Alicia (1983).

If we focus on the past 20 years (1999-2018), there have been 11 major hurricane landfalls using the $V_{\max }$ definition, with none from Georgia to Maine. If the MSLP definition were used, 19 major hurricanes made landfall in the past 20 years, including 5 from Georgia to Maine. All 5 of these caused at least $\$ 5$ billion in normalized damage, and 4 of them caused at least $\$ 10$ billion in normalized damage. Of the 61 hurricanes that caused $\geq \$ 5$ billion in damage, $69 \%$ are classified as major using $V_{\max }$, while $84 \%$ are classified as major using MSLP. While more hurricanes are classified as major using our MSLP threshold, we argue that these additional storms have caused damage similar to that of the median major hurricane using the $V_{\max }$ threshold. These storms warrant being classified as major hurricanes given their overall intensity (as measured by MSLP).

While hurricanes cause fatalities in many ways, $\sim 50 \%$ of fatalities from 1963-2012 were caused by storm surge, while $25 \%$ were caused by freshwater floods and mudslides. Examining direct fatalities from CONUS landfalling hurricanes from 1988-2018, MSLP and direct fatalities $\left(r_{\text {rank }}=0.61\right)$ have a much stronger correlation than $V_{\max }$ and direct fatalities $\left(r_{\text {rank }}=0.33\right)$. This makes physical sense given the improved relationship between MSLP and storm size. 
$V_{\max }$ partially describes the overall wind threat, but it provides less information on the overall storm risks than does MSLP. We note that $V_{\max }$ remains a very useful measure for understanding hurricane intensity, however. For example, in the insurance industry, $V_{\max }$ and its speed of decay while over land, plus the type of terrain over which a storm crosses, remain very important components in loss modeling.

MSLP, on the other hand, is strongly correlated with both $V_{\max }$ and storm size, which is directly related to storm surge as well as a larger wind and rain footprint. MSLP also is more skillful than $V_{\max }$ in predicting fatalities in CONUS landfalling hurricanes (for 19882018). Not surprisingly, insurance industry catastrophe models dealing with wind and/ or storm surge-modeled scenarios include mathematical formulations that seek to interpret surface wind and pressure relationships. To determine actual losses, these models further incorporate MSLP/ $V_{\max }$ with other hazard parameters. Beyond catastrophe modeling, the insurance industry uses MSLP as a potential trigger for specifically placed policies or catastrophe bonds.

\section{Implications}

We have examined 165 CONUS hurricane landfalls from 1900-2018 in order to determine the relationship between hurricane intensity and risk. Hurricane intensity can be expressed as MSLP or $V_{\max }$, and risk can be measured as damage (in dollars) or fatalities. Traditionally, boundaries of hurricane categories have been defined by $V_{\max }$.

We have shown that MSLP serves as a better predictor of damage over a span of 118 years $\left(r_{\text {rank }}=0.77\right)$. Even more, the relationship between MSLP and damage is stronger for the last 11 years $\left(r_{\text {rank }}=0.89\right)$. Additionally, we have also shown that hurricane fatalities correlate more strongly with MSLP.

Given that the Saffir-Simpson scale was developed to characterize the risk of hurricanes to the public and that MSLP can be measured more accurately, we propose classifying future hurricanes using MSLP as the primary metric. While a revised scale could still include ranges of wind speeds for each category, aligning the boundaries of such a scale with pressure will better inform the public of the true risk of a hurricane.

\section{$\equiv$ METADATA}

BAMS: What would you like readers to learn from this article?

Phil Klotzbach (Colorado State University):

While the Saffir-Simpson Hurricane Wind Scale serves as a useful tool for conveying part of the wind risk caused by hurricanes, surface pressure is a more robust metric characterizing the overall intensity of a hurricane (and consequently risk to both life and property).

BAMS: How did you become interested in the topic of this article?

\begin{abstract}
PK: When looking at aircraft reconnaissance data, my first inclination is to look at the pressure data as opposed to wind data. In almost all cases, if the pressure is dropping, the storm's winds are increasing, the storm is getting larger, or potentially a combination of both factors. If the wind is decreasing but the pressure is still dropping, you likely have a storm that is getting larger-which still means it is extremely dangerous. I was curious to see how well pressure worked as a predictor of damage using a long-term dataset, especially because pressure is much easier to measure than wind.
\end{abstract}

BAMS: What surprised you in this study?

PK: I was quite surprised to see how well pressure worked as a predictor of historical normalized damage. Pressure accounted for $60 \%$ of the variability in damage, without even accounting for the population impacted by the hurricane. Hurricanes making landfall in more densely populated areas are going to cause more damage than hurricanes making landfall in sparsely populated areas, with all else being equal.

BAMS: What was the biggest challenge in this project?

PK: We had considerable discussion as to how exactly we wanted to assign pressure values to each category of the Saffir-Simpson Scale. I was happy that our approach using lifetime maximum intensities ended up with pressure values that matched up quite closely with what was originally in the scale. 
AMS titles now

available as eBooks

at springer.com

\section{AMS BOOKS}

\section{RESEARCH APPLICATIONS HISTORY}

www.ametsoc.org/amsbookstore

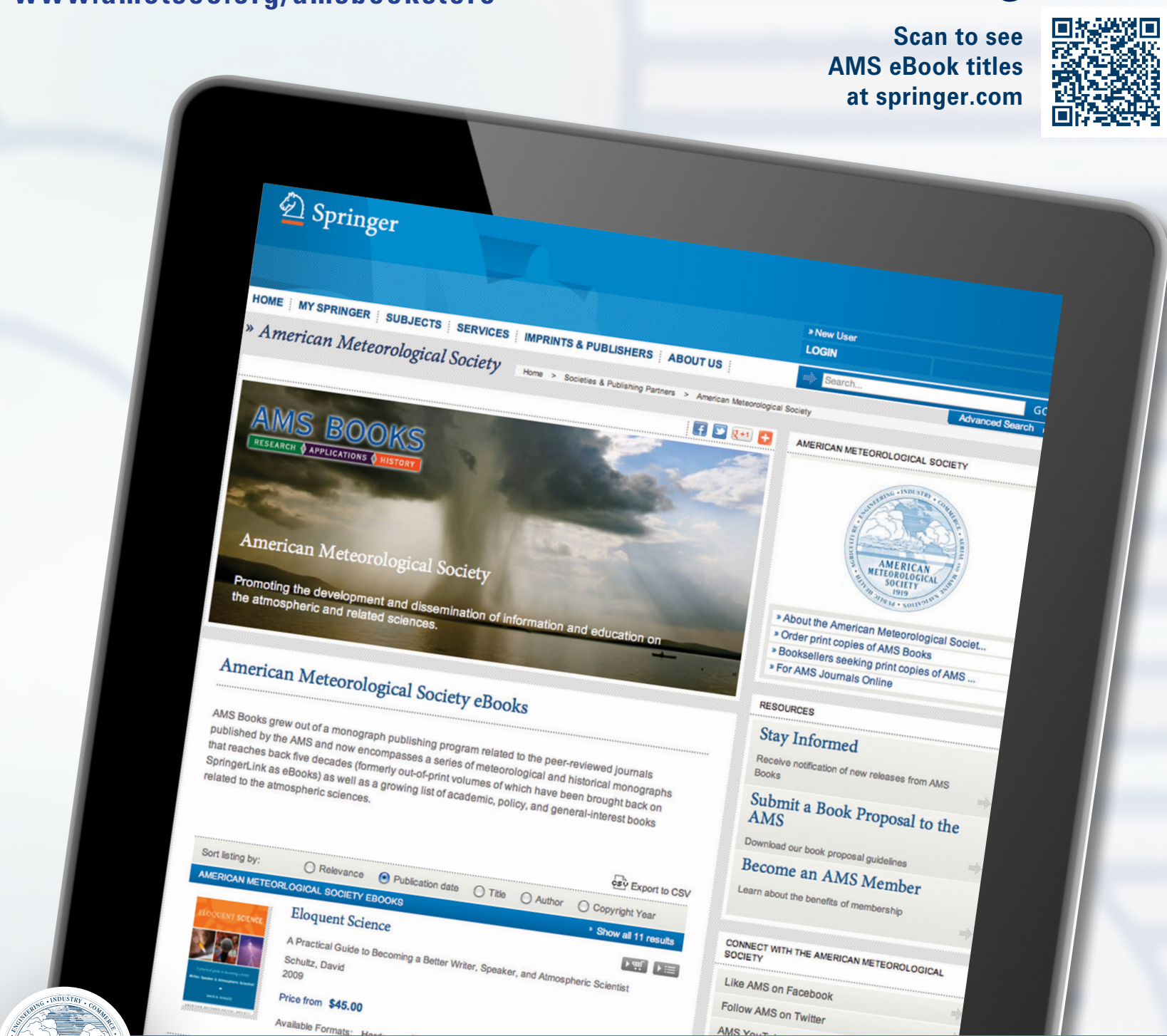

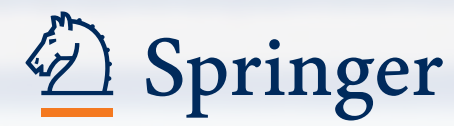

A M E R I C A N M E T EOROLO G I A L S O I E T Y 